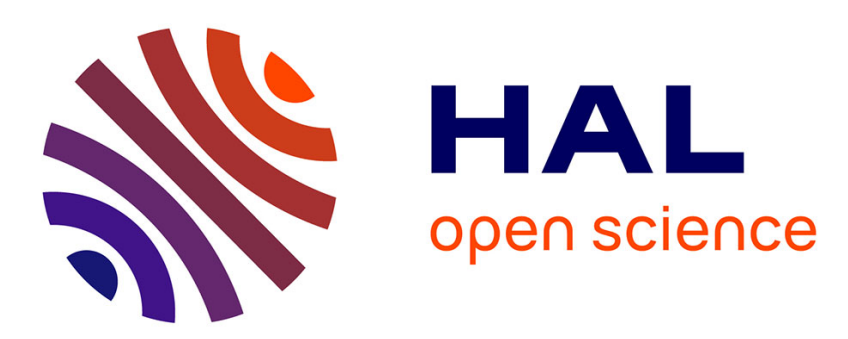

\title{
L'environnementalisme social mexicain? Une version endémique de l'écologie politique
}

\author{
Jean Foyer, David Dumoulin Kervran
}

\section{To cite this version:}

Jean Foyer, David Dumoulin Kervran. L'environnementalisme social mexicain? Une version endémique de l'écologie politique. Écologie \& politique: sciences, culture, société, 2013, Les écologies politiques aujourd'hui (4), Amérique latine, 46, pp.83-94. 10.3917/ecopo.046.0083 . halshs-01542359

\section{HAL Id: halshs-01542359 \\ https://shs.hal.science/halshs-01542359}

Submitted on 22 Jun 2017

HAL is a multi-disciplinary open access archive for the deposit and dissemination of scientific research documents, whether they are published or not. The documents may come from teaching and research institutions in France or abroad, or from public or private research centers.
L'archive ouverte pluridisciplinaire HAL, est destinée au dépôt et à la diffusion de documents scientifiques de niveau recherche, publiés ou non, émanant des établissements d'enseignement et de recherche français ou étrangers, des laboratoires publics ou privés. 


\title{
L'environnementalisme social mexicain: une version endémique de l'écologie politique
}

\author{
Jean Foyer et David Dumoulin
}

\begin{abstract}
Résumé - Cet article a pour objectif de présenter une analyse de l'une des composantes les plus créatives de l'écologie politique mexicaine que l'on qualifie d'environnementalisme social. Il s'agit d'historiciser ici ce courant hétéroclite en montrant comment il a émergé dans les années 1970 et 1980 sur la base de théories critiques, de mouvements politiques issus de la gauche mexicaine et de projets de développement local. Les années 1990 et 2000 voient ce mouvement de l'environnementalisme social confronté au double défi de l'institutionnalisation et de l'internationalisation. L'institutionnalisation pose la question délicate du rapport à l'État mexicain et à sa tradition clientéliste, quand l'internationalisation marque l'arrivée de nouvelles sources de financements et de nouveaux acteurs qui tendent à aligner l'écologie politique mexicaine sur des problématiques globales et des modes d'intervention relevant de l'expertise critique.
\end{abstract}

Mots CLÉs - Environnementalisme social, Mexique, développement durable, communauté, expertise.

ABstRACt - The objective of this article is to present an analysis of one of the most creative currents of Mexican political ecology that we will refer to here as social environmentalism. We will present its historical context by showing how it began at the crossroads of critical theory: between left-wing Mexican political movements and local development projects. During the last two decades, the social environmental movement has faced two principal challenges: institutionalization and internationalization. Institutionalization refers to the sensitive question of the movements relationship to the Mexican state and its tradition of clientelism. Internationalization pertains to the arrival of new funding sources and actors that tend to orient political ecology toward global issues and methodologies based on critical expertise.

KEYWORDS - Social environmentalism, Mexico, sustainable development, local communities, experts.

Au Mexique, le vocable d'écologie politique fait référence à un ensemble hétéroclite de mobilisations environnementales et politiques allant des mouvements locaux de résistances à des projets de construction d'infrastructures (hydrauliques, minières, nucléaires, pétrolières, touristiques, etc.) ${ }^{2}$ aux très diverses mobilisations à caractère environnemental des communautés indigènes $^{3}$, en passant par l'important courant d'écologie urbaine qui s'est constitué

1. Cette recherche a reçu le soutien du programme «Environmental Governance in Latin America and the Caribbean: Developing Sustainable and Equitable Approaches to Natural Resource Use», ENGOV (CEE, Socio-economic Sciences and the Humanities, FP7-SSH-2010-3).

2. Récemment, l'opposition à un vaste projet minier sur le territoire de Wirikuta, lieu de pèlerinage des Huichols, représente ce type de mobilisation.

3. Victor Manuel Toledo recense de nombreuses initiatives impliquant une dimension environnementale en territoires indigènes et parle ainsi d'un «zapatisme vert» à propos de cette rencontre entre populations indigènes et écologie. V. M. Toledo, La Paz en Chiapas. Ecología, luchas indígenas y modernidad alternativa, Quinto Sol, Mexico, 2000. 
face aux problèmes de pollutions dans le cadre du développement incontrôlé des mégapoles mexicaines. Il existe en fait une myriade de mobilisations collectives locales de nature environnementale. On peut même penser que le caractère très localisé de ces mobilisations est un trait essentiel des mouvements sociaux et environnementaux mexicains ${ }^{4}$.

Sans nier l'importance de ces mobilisations, plus ou moins ponctuelles et locales, nous préférons cependant centrer nos analyses sur un courant plus restreint de l'écologie politique mais probablement plus structuré et plus clairement identifiable à l'écologie politique que les précédents du fait de son souci constant de lier les problématiques environnementales aux problématiques sociales, au-delà de luttes ponctuelles. Pour bien le démarquer d'une approche plus conservationniste ${ }^{5}$ et souligner la centralité du lien entre logiques environnementales et sociales, nous l'avons nommé «environnementalisme social $^{6}{ }$. L'écologie urbaine, depuis les luttes au sein de la capitale à la fin des années 1980 jusqu'aux combats actuels à la frontière nord, a constitué un autre courant qui n'est que partiellement connecté à cette mouvance rurale. Nous avons aussi choisi de nous intéresser à ce courant car il est particulièrement pertinent pour montrer les modes mexicains d'appropriation de l'écologie politique. Cependant, le Mexique n'est pas qu'un simple récepteur de concepts et de pratiques écologiques importés depuis l'international, c'est aussi un lieu où il s'en crée et le courant de l'environnementalisme social en est une des illustrations les plus nettes. Un autre intérêt de cet environnementalisme social mexicain est qu'il regroupe des acteurs issus du monde académique, des ONG et des organisations locales paysannes et/ou indigènes. Il est donc aussi intéressant pour souligner la circulation des idées et des pratiques de l'écologie politique entre ces différentes catégories d'acteurs.

Il s'agira dans cet article de présenter la trajectoire historique de ce courant en montrant tout d'abord son émergence dans les années 1970 et 1980 à l'interface de théories critiques, de mouvements politiques issus de la gauche mexicaine et de projets de développement local. Nous montrerons ensuite comment, dans les années 1990 et 2000, il s'est transformé et peu à peu recomposé sous l'influence de deux dynamiques: celle de son institutionnalisation et celles de son internationalisation.

\footnotetext{
4. M. A. Velazquez Garcia, «Los movimientos ambientales en Mexico», in I. Bizberg et F. Zapata (dir.), Los Movimientos Sociales, El Colegio de Mexico, Mexico, 2010 ; G. Pleyers, «Le réinvestissement de l'espace local par les mouvements mexicains : refuge après les impasses politiques ou creuset d'une nouvelle culture politique ?», Cahiers des Amériques latines, n 66, 2011, p. 39-56.

5. Pour une histoire de l'environnementalisme au Mexique, et notamment les différents mouvements de conservation de la nature, voir L. Simonian, La defensa de la tierra del jaguar : una historia de la conservación en Mexico, CONABIO \& INE-SEMARNAP, Mexico, 1999.

6. Le terme d'environnementalisme social n'est pas utilisé par les acteurs analysés ici. Il renvoie à une catégorie d'analyse proposée en premier lieu dans A. Gonzalez Martinez, «Socio-Ecological Struggles in Mexico: The Prospects », International Journal of Sociology and Social Policy, vol. 12, $\mathrm{n}^{\circ}$ 4-7, 1992, p. 113-128.
} 


\section{Le cocktail original de l'environnementalisme social mexicain: théories critiques, engagement politique et développement communautaire}

Dans la deuxième moitié des années 1970 et tout au long des années 1980 va émerger au Mexique un courant environnementaliste fortement ancré dans le tissu social, et dont le cœur de l'activité repose davantage sur la problématique du développement communautaire en milieu rural que sur la conservation de la nature ${ }^{7}$. Déjà, dès 1972, la création du Centro de Ecodesarrollo montre le rapprochement entre la problématique du développement et celle de l'environnement, ainsi que l'influence d'Ivan Illich, qui a animé le Centre interculturel de documentation au Mexique entre 1961 et 1976, et d'une théorie comme celle de l'écodéveloppement d'Ignacy Sachs. Cette première initiative montre aussi l'importance du monde universitaire dans la création et les activités de ce type d'organisations. Le mouvement étudiant de 1968, malgré la très dure répression dont il a fait l'objet, a engendré une dynamique d'organisation sociale et une volonté de participation qui s'est traduite par un souci d'implication de la recherche et des étudiants dans les transformations sociopolitiques du pays. Cette volonté traverse tous les différents départements disciplinaires des universités publiques mexicaines, depuis les sciences sociales jusqu'aux sciences de la vie, qui se politisent très clairement sous l'influence des différents courants de pensée souvent issus du marxisme.

Les membres fondateurs de l'une des premières ONG explicitement environnementaliste, le Grupo de Estudios Ambientales (GEA), sont ainsi marqués par les thèses sur le municipalisme libertaire, entre anarcho-communisme et écologie radicale, du penseur américain Murray Bookchin ${ }^{8}$, mais aussi et surtout par la personnalité et la pensée plus proprement mexicaine du professeur d'agronomie Efraím Hernandez Xolocotzi qui enseigne alors à l'université de Chapingo 9 . Celui-ci prône une "ciencia de huarache» (en sandale), c'est-àdire une science de terrain, basée sur le contact direct avec les populations rurales et la reconnaissance des savoirs et pratiques traditionnels, et portée par le courant très dynamique des recherches ethnobotaniques. Ce positionnement méthodologique et épistémologique s'accommode très bien des aspirations politiques d'engagement auprès des populations locales, et les futurs membres de GEA vont alors expérimenter la vie paysanne dans le municipe de Xalapa, Veracruz, aux alentours de 1975. Ce n'est qu'en 1977 que GEA se constitue formellement comme association civile autour de ces étudiants issus de diverses disciplines, depuis l'anthropologie jusqu'à la biologie, en passant par l'agronomie. Ils sont tout d'abord actifs dans la Sierra Norte de Puebla et

7. D. Carruthers, «Indigenous Ecology and the Politics of Linkage in Mexican Social Movements», Third World Quaterly, vol. 17, n 5, 1996, p. 1007-1028.

8. P. Dieuaide, «Le municipalisme libertaire de Murray Bookchin», Ecorev', n² 21, 2005, <ecorev. org>.

9. Diaz de Leon, entretien personnel réalisé le 22 janvier 2006. 
dans les montagnes du Guerrero autour de l'une des toutes premières expériences de foresterie communautaire, mais aussi dans le sud de Mexico (Chinampas de Xochimilco), avec un projet de récupération du maïs traditionnel. Entre recherche alternative, formation des populations locales et montage de projets de développement au niveau des communautés, GEA se démarque d'un développement communautaire classique par la place accordée à la perspective environnementaliste.

Un second groupe important est constitué de chercheurs de l'université nationale autonome de Mexico (UNAM), essentiellement des biologistes engagés dans l'opposition de gauche au Parti révolutionnaire institutionnel (PRI). Les deux figures centrales de ce groupe sont alors l'ethnobotaniste Victor Manuel Toledo, qui essaie à cette époque d'opérer une synthèse théorique entre marxisme et écologie ${ }^{10}$, et la biologiste Julia Carabias, à l'origine d'un groupe de réflexion sur le lien entre questions environnementales et développement rural au sein du Parti socialiste unifié du Mexique (PSUM). Ce parti, issu de la fusion du Parti communiste mexicain avec différents courants de la gauche mexicaine, a remporté les élections municipales de Alcauzauca, dans l'État de Guerrero, à la toute fin des années 1970. Cette victoire va fournir l'occasion pour ce groupe de passer de la réflexion à l'action politique et de mettre en place un projet expérimental qui constituait alors une expérience originale de développement environnemental dans cette région. Il s'agissait essentiellement d'établir un diagnostic environnemental des ressources naturelles et, sur cette base, d'élaborer un plan de gestion au niveau municipal. Cette expérience, avec sa méthodologie alors très novatrice, est considérée comme un succès. Elle va prendre de l'ampleur à travers le Programa de Aprovechamiento Integral de Recursos Naturales (PAIR) qui s'étend dans différentes régions du pays, notamment sur la Meseta Purépecha du Michoacán, dans la Chinantla de Oaxaca et dans la région semi-désertique de Durango ${ }^{11}$.

Ce type d'expériences alternatives de développement communautaire se développe également au niveau local, avec par exemple l'organisation Estudios Rurales y Asesoria (ERA) qui s'installe dans l'État d'Oaxaca dans la deuxième moitié des années 1980. ERA participe alors au mouvement de récupération par les communautés de la Sierra de Juarez de leurs droits sur leur territoire forestier contre les concessions accordées à des entreprises parapubliques. Cette première mobilisation sera au fondement de l'une des plus intéressantes expériences de foresterie communautaire à travers la formation de l'Unión Zapoteca-Chinanteca (UZACHI), une organisation intercommunautaire qui vise à exploiter durablement ses ressources forestières.

10. V. M. Toledo, «La otra guerra florida», Nexos, $\mathrm{n}^{\circ}$ 6, septembre, 1983.

11. J. Carabias, E. Provencio et C. Toledo (dir.), Manejo de recursos naturales y pobreza rural, Fondo de Cultura Económica \& UNAM, Mexico, 1994. 
Outre les diverses expériences de foresterie communautaire qui se développent à partir de la fin des années $1980^{12}$, en particulier à partir du Plan d'action forestier tropical (1982), et qui deviendront un courant essentiel de cet environnementalisme social jusqu'à aujourd'hui, rappelons que c'est à ce même moment que la caféiculture biologique prend son essor sous l'impulsion du travail de base des courants progressistes de l'Église catholique qui favorisent l'organisation de coopératives de petits producteurs de café, notamment dans les États d'Oaxaca et du Chiapas.

Au début des années 1990, à la suite du Sommet de la Terre de Rio, une deuxième génération d'organisations de ce type apparaît avec, pour ne citer que quelques exemples représentatifs, le Grupo Interdisciplinario de tecnologia Rural Apropiada (GIRA) qui opère dans les communautés indigènes du Michoacán, le Proyecto Sierra de Santa Marta (PSSM) dans le sud de l'État du Veracruz ou encore le Grupo GAIA sur la côte du Oaxaca. Ces organisations promeuvent l'éducation environnementale, elles cherchent également à renforcer l'organisation communautaire sur diverses thématiques environnementales (sensibilisation à la problématique de la déforestation, rationalisation de l'usage de l'eau, etc.) à travers des ateliers de formation. Elles proposent également de mettre en place un nouvel ordonnancement du territoire en établissant en concertation avec les communautés des zones d'usages spécifiques des ressources naturelles. Elles tentent également de développer des initiatives alternatives de production (bassin de pisciculture, diversification agricole, utilisation d'engrais verts, lombricompost...), un écotourisme léger (chez l'habitant ou dans des «cabanas») ou encore l'établissement de réserves naturelles communautaires destinées à la conservation des ressources naturelles locales (forestières et aquatiques notamment).

Au début des années 1990, l'environnementalisme social mexicain prend donc la forme de divers projets de développement communautaires où des assesseurs issus du monde universitaire ou des courants religieux progressistes essaient de mettre en œuvre des formes alternatives de mise en valeur des ressources naturelles, sur la base d'organisations communautaires locales. Si l'environnementalisme social ne vient que rarement d'une dynamique communautaire endogène, il cherche systématiquement à s'appuyer sur les organisations et les communautés locales. Bien que ces diverses expériences soient souvent novatrices, leur coordination reste faible malgré l'existence, dès 1984, du REDE (Réseau d'écologie) qui mettait en lien quelque deux cents individus et groupes citoyens autour d'expériences locales d'écodéveloppement ${ }^{13}$. En organisant la première rencontre nationale des groupes écologistes qui réunit environ 300 organisations, ce réseau marque à cette époque une des premières tentatives d'alliance au sein de l'environnementalisme mexicain. Les occasions d'alliances plus ou moins durables et hétéroclites où se retrouvent les acteurs

12. D. Barton Bray et L. Merino Perez, La experiencia de las comunidades forestales en Mexico, INE-SEMARNAT, Mexico, 2004.

13. A. Gonzalez Martinez, op. cit. 
de l'environnementalisme social au côté de courants plus urbains et, moins souvent, des conservationnistes s'intensifient alors: le grand tremblement de terre de 1985, la résistance face à des méga-projets de barrage ou d'exploitation forestière, la tentative d'unification du mouvement environnemental dans la Federación Conservacionista Mexicana (FECOMEX) en 1985 ou la mobilisation antinucléaire comme celle de la Coordinadora Nacional Contra Laguna Verde (CONCLAVE) contre la centrale de Laguna Verde à partir de 1988, puis aussi les négociations onusiennes de Rio en 1992 et celles de l'Accord de libre-échange nord-américain (ALENA). Toutes ces alliances permettent l'émergence progressive d'un agenda commun multisectoriel, mais elles sont fragiles et ont de la peine à s'inscrire dans la durée ou à maintenir des positions vraiment cohérentes tant leurs membres sont divers, les positions idéologiques sensibles et les stratégies différentes. Après cette période d'expérimentation et de montée de la contestation, les années 1990 vont marquer une certaine forme de maturation du mouvement où des choix stratégiques vont s'opérer sur deux points notamment : celui du rapport à l'État d'une part, celui de l'articulation à l'international d'autre part.

\section{L'institutionnalisation ou le dilemme mexicain du rapport à l'État}

On doit replacer l'essor des ONG environnementales mexicaines en général dans le contexte national plus large de démocratisation et d'ouverture du système mexicain, qui laisse une marge de manœuvre pour certaines expériences en dehors du corporatisme officiel ${ }^{14}$. Néanmoins, dans un État contrôlé pendant près de soixante-dix ans par un parti, le PRI ${ }^{15}$, marqué par son corporatisme et par les réseaux clientélistes qu'il entretient, la question du rapport aux institutions officielles représente un dilemme fondamental pour l'ensemble des mouvements sociaux mexicains. Ce dilemme se pose de la manière suivante: maintenir son indépendance au risque de se couper de tous leviers institutionnels et de toute ressource, ou bien accéder à des postes et à des moyens (financiers notamment) beaucoup plus importants au risque de légitimer le système mis en place par le PRI et d'en subir les nombreuses contraintes.

À partir du milieu des années 1990, le groupe qui s'est constitué autour de Julia Carabias à l'origine du PAIR va faire le choix de l'institutionnalisation, puisqu'au moment de l'arrivée d'Ernesto Zedillo au pouvoir en 1994, l'opportunité se présente de monter le premier ministère de l'Environnement mexicain, le SEMARNAP ${ }^{16}$. Cette opportunité correspond également au verdissement

14. E. Mollard et E. Lopez, «L'instrumentalisation des ONG au Mexique en situation indienne et non indienne», Autrepart, $\mathrm{n}^{\circ}$ 37, 2006. p. 129-146.

15. Le PRI a contrôlé le Mexique de la fin de la révolution mexicaine, dans les années 1920, à 2000. Après une transition de douze ans marquée par la présidence du parti de droite, le Parti action nationale (PAN), le PRI a repris le pouvoir au niveau national en 2012, ainsi que dans de nombreux États.

16. Secretaria de Medio Ambiente, Recursos Naturales y Pesca qui, quand le secteur de la pêche lui sera enlevé, à la fin du sexennat de Carabias à la tête du ministère, deviendra le Secretaria de Medio Ambiente y Recursos Naturales (SEMARNAT). 
du gouvernement mexicain à la fin des années 1980 face à la pression interne, notamment pour canaliser les nombreux mouvements environnementalistes qui émergent à cette même époque comme une force contestataire. L'intégration de l'équipe Carabias dans le parti-État peut donc aussi se lire dans la grande tradition mexicaine de cooptation des mouvements sociaux. Julia Carabias, après un moment d'hésitation, accepte la proposition à condition d'entrer au gouvernement avec son équipe complète aux postes clés du nouveau ministère de l'Environnement. À partir du concept revendiqué de développement durable, cette équipe va mener durant six ans un travail considérable de consolidation du cadre institutionnel et légal de l'environnementalisme officiel mexicain, opérer un travail de formation des cadres administratifs aux thématiques environnementales et multiplier et renforcer le contrôle des aires naturelles protégées, tout en considérant les activités humaines ${ }^{17}$. Même si les critiques des différents mouvements sociaux ont été extrêmement virulentes contre la personne de Carabias, notamment pour sa ligne jugée trop conservationnistes et plus encore pour ses prises de positions contre le mouvement néozapatiste du Chiapas, le sexennat de Julia Carabias représente un bon quantitatif et qualitatif sans précédent dans la prise en compte des problématiques environnementales au Mexique. Malgré le tournant plus conservationniste des ministères panistes à partir des années 2000, l'opportunité de participer à la direction des programmes les plus sociaux hérités de l'administration Carabias va également tenter d'autres figures de l'environnementalisme social au cours des années 2000, avec l'idée de diffuser des expériences de gestions locales de foresterie communautaire ou de mise en valeur des ressources naturelles en zone indigène. Ce passage des ONG aux institutions et du local au national s'avère toujours périlleux. Si les budgets gérés et les instruments de mise en œuvre sont sans commune mesure avec ceux de la société civile, ces moyens restent dérisoires par rapport à d'autres politiques publiques, agricoles par exemple, beaucoup plus structurelles et prédatrices au niveau environnemental.

Un membre du GEA explique ces difficultés de traduction des initiatives civiles en politiques publique:

Nous travaillons toujours avec une certaine marge d'indépendance parce que nous ne croyons pas en l'État et nous ne voulons pas appuyer ses politiques néolibérales. Mais il faut qu'il y ait parfois des instances de négociations ouvertes pour pouvoir faire usage de certaines ressources ou avoir de l'influence. Parce que si tu n'entres pas, si tu ne négocies pas, ils te marginalisent et c'est important de se positionner politiquement, bien que nous ne soyons liés à aucun pouvoir de l'État ni à aucun $\operatorname{parti}^{18}$.

Malgré certains discours critiques qui pourraient faire apparaître un rejet en bloc de l'État, la position face à ce dernier est plus subtile et semble admettre une certaine marge de manœuvre à travers des collaborations avec les institutions environnementales. Au gré des changements de personnels dans les

17. Julia Carabias, entretien personnel réalisé le 14 octobre 2004.

18. Diaz de Leon, entretien personnel réalisé le 22 janvier 2006. 
bureaux centraux du SEMARNAT et aussi des différents États, des relations avec des membres des instances gouvernementales, des nouveaux programmes et des possibilités de financements, des brèches ou «fenêtres d'opportunité » s'ouvrent parfois pour les membres de l'environnementalisme social.

Pourtant, face à cette approche plus pragmatique, une troisième ligne plus radicale se développe notamment à partir de l'insurrection zapatiste de 1994. Des organisations qui viennent s'implanter au Chiapas, comme Maderas del Pueblo del Sureste ou le CIEPAC (Centro de Investigaciones Economicas y Politicas de Accion Comunitaria), dénoncent différentes initiatives environnementales du gouvernement mexicain comme les projets de replacement de populations locales en dehors de la réserve de la biosphère de Montes Azules, des projets de bioprospection comme l'ICBG Maya ${ }^{19}$, des projets d'écotourisme de luxe ou encore une initiative comme le corridor biologique mésoaméricain (CBM), considérée comme le versant environnemental du plan d'intégration commerciale régionale Puebla-Panama. Se revendiquant d'une approche autonomiste zapatiste en rupture frontale avec le gouvernement, ces organisations s'éloignent des activités de développement communautaire pour mener des campagnes de dénonciation contre l'environnementalisme officiel, jugé trop conservationniste ou servant les intérêts des firmes multinationales.

Le positionnement face à l'État mexicain représente donc un point central dans l'évolution de l'environnementalisme social mexicain. De la reconversion dans les institutions environnementales mexicaines à leur critique radicale, en passant par une position intermédiaire et pragmatique de collaboration sélective, cette question du rapport à l'État contribue à reconfigurer largement l'environnementalisme mexicain.

\section{Internationalisation des financements et décrochage du local}

En jouant un rôle peut-être marginal mais néanmoins pionnier, les organisations de l'environnementalisme social sont bien placées pour recevoir, à partir de 1992, l'afflux de financement en provenance de l'international sur les thématiques du développement durable et de la biodiversité, après la période des années 1980 où la plupart d'entre elles étaient financées sur fonds propres ou, plus rarement, grâce à l'argent public. Là encore, on constate une certaine similitude dans les canaux de financements internationaux. On note d'abord une omniprésence de la Fondation Ford (également de la Mc Arthur et de la Friedrich Ebert), presque systématiquement présente dans l'appui à ces organisations, au moins jusqu'à la fin des années 1990. Ensuite, on retrouve souvent l'organisation de coopération hollandaise NOVIB, mais aussi les organisations religieuses allemandes comme Misereor ou Pan Por el Mundo. La coopération des pays du nord de l'Europe (Scandinavie et Allemagne notamment,

19. D. Dumoulin et J. Foyer, «ONG transnationales et experts dans le débat démocratique: bioprospection et savoirs indigènes au Mexique », Problèmes d'Amérique latine, ${ }^{\circ} 54$, automne, 2004 , p. $95-122$. 
mais aussi l'Angleterre et son ministère pour le Développement international, DFID) et le Fonds pour l'environnement mondial (FEM) de la Banque mondiale représentent aussi des sources de financements importantes pour ces acteurs, qui leur permettent de se consolider et, à d'autres, de créer de nouvelles organisations.

On peut cependant se demander si la dépendance structurelle vis-à-vis des fonds institutionnels internationaux (mais aussi nationaux comme ceux distribués par le Fondo Mexicano para la Conservacion de la Naturaleza) n'a pas contribué à saper la dimension militante et alternative des organisations du développement communautaire, pour les transformer en prestataires de service et en opérateurs techniques locaux. L'exemple de l'organisation PSSM est ainsi significatif. L'organisation finit par manifester en effet une telle proximité avec les différentes institutions de la réserve de la biosphère de Los Tuxtlas, qu'il s'agisse de sa participation au dessin d'ordonnancement écologique, à ses instances de gouvernance ou dans l'opération de certains de ses programmes, qu'on peut légitimement se demander si elle ne fait pas partie intégrante du dispositif officiel. Dans un contexte national et international où les thématiques environnementales sont institutionnalisées, le choix des thèmes de travail est en partie déterminé par les opportunités de financement. Luisa Paré, fondatrice de cette organisation et militante environnementale depuis plus de vingt ans, fait l'analyse suivante: «Nous sommes passés d'une époque militante, comme dans le cas de la Sierra Norte d'Oaxaca, avec un fort engagement politique de ceux qui allaient vivre et travailler dans les communautés, à, aujourd'hui, une attitude plus professionnelle, où les gens n'essaient plus vraiment de changer les choses et privilégient la dimension technique de leur travail. Je ne juge pas, je dis juste que ce sont des moments et des formes d'action différents ${ }^{20}$.»

L'arrivée de fonds étrangers permet donc une professionnalisation de certaines organisations, ainsi que l'adoption de lignes de travail plus générales qui ne sont plus tant liées à la promotion de projets de développement local qu'à des questions de stratégies et de positionnements politiques (expertise, lobbying, campagnes...) au niveau national ou international. Au niveau national se forment ainsi à Mexico deux organisations importantes chargées, elles aussi, de produire une certaine expertise à la fois technique et politique sur des questions environnementales liées au monde rural : le Centro de Estudios para el Cambio en el Campo Mexicano (CECCAM) et le Consejo Civil Mexicano para la Silvicultura Sostenible (CCMSS). Le CECCAM se veut dans un premier temps un centre de réflexion critique qui produit une réflexion politique pour un syndicat paysan formé depuis 1985, l'Unión Nacional de Organizaciones Regionales Campesinas Autónomas (UNORCA), qui appartient à la Via Campesina. Si son champ d'analyse se veut tout d'abord tourné vers la problématique rurale, le CECCAM, à travers le traitement de problèmes liés à la foresterie, à l'agroécologie ou aux biotechnologies, produit un discours qui tend à mettre

20. Entretien personnel réalisé le 3 octobre 2008. 
en évidence la pénétration progressive des thématiques environnementales dans le monde des organisations rurales. À l'initiative d'organisations comme GEA, ERA ou GIRA, le CCMSS est, quant à lui, formé quelques années plus tard au milieu des années 1990 pour essayer de capitaliser différentes expériences locales et promouvoir la foresterie communautaire au niveau national, notamment devant les pouvoirs publics. Toutes ces différentes organisations, et plus particulièrement encore celles qui ont une perspective nationale comme le CECCAM et le CCMSS, montrent une certaine maturation de ces organisations civiles mexicaines à mi-chemin entre expertise technique et activité militante, mais aussi entre développement rural et environnementalisme. Les organisations comme le CECCAM ou le CCMSS marquent aussi un tournant qualitatif avec le passage d'activités de développement à des activités de coordination et d'influence politique sur les institutions et l'opinion, qui les rapprochent des organisations d'experts militants et des campainers. Ces évolutions correspondent aussi à l'arrimage de ces organisations aux réseaux transnationaux, ainsi qu'à l'arrivée de nouveaux acteurs externes.

La deuxième partie des années 1990 et le début des années 2000 correspondent en effet à l'arrivée d'acteurs transnationaux qui vont influer sur le paysage déjà dense de l'environnementalisme social mexicain. Catherine Marielle, du GEA, résume en ces termes ce qu'elle considère comme une nouvelle étape:

L'arrivée de Greenpeace et ETC Group est beaucoup plus récente. À la différence de ces organisations, nous n'avons pas le temps de travailler véritablement la visibilité comme le fait Greenpeace dont c'est la stratégie pour récolter des fonds. Il s'agit d'une étape très distincte, très nouvelle, avec ses propres caractéristiques qui correspondent à la globalisation. Ces organisations sont arrivées après Rio 92. Avant, notre travail se situait beaucoup plus au niveau national alors que nous participons désormais à des réseaux internationaux ${ }^{21}$.

Si Greenpeace était présente au Mexique depuis 1993 à travers des campagnes liées à la pollution atmosphérique de Mexico, à la centrale nucléaire de Laguna Verde ou encore à l'importation de déchets toxiques, ce n'est qu'en 1998 qu'elle décide d'ouvrir une campagne «Ingénierie génétique» au Mexique. Ce pays est considéré comme une zone stratégique du fait de sa biodiversité agricole et du risque que représentent les importations américaines. Un des objectifs est aussi de surveiller et de renforcer si possible le moratoire qui est alors déclaré par le gouvernement mexicain sur le maïs transgénique $^{22}$ et d'empêcher, par des actions militantes et légales notamment, l'autorisation des maïs transgéniques mexicains. En 1999, la représentante de la RAFI (Rural Advancement Foundation International, qui, en 2001, sera renommée ETC Group), Silvia Ribeiro, venue d'Uruguay avec une expérience de journalisme militant, arrive au Mexique. Malgré l'effectif restreint de ces organisations, Greenpeace et ETC Group vont jouer un rôle central dans le lancement et l'orchestration des campagnes contre la bioprospection et contre

21. Entretien personnel réalisé le 22 janvier 2006.

22. Gustavo Ampugnani, entretien personnel réalisé le 23 janvier 2006. 
le maïs transgénique, notamment en important au Mexique certaines méthodes communicationnelles et en devenant des points nodaux du réseau d'opposition alors en cours de formation. Ils créent très vite des alliances avec les organisations environnementales et paysannes mexicaines. Ainsi, Greenpeace se lie au GEA et à l'ANEC (autre syndicat paysan de la Via Campesina) pour fonder le mouvement Sin Maíz No Hay País (Sans maïs, pas de pays) quand ETC Group se montre plus proche du CECCAM et de l'UNORCA, et forme la Red en defensa del Maíz. De manière générale, l'arrivée de ces organisations internationales dans le contexte de la globalisation semble contribuer à «tirer» une partie des acteurs de l'environnementalisme social mexicain du local vers le mondial et de problématiques concrètes ancrées dans les communautés locales à des problématiques plus structurelles définies en termes de politiques nationales ou internationales. Avec cette modification dans la perception des échelles, une partie de l'environnementalisme mexicain est attirée par la reconfiguration idéologique que représente l'opposition à la globalisation libérale. Cette reconfiguration idéologique se manifeste dans la dénonciation systématique des accords commerciaux de l'ALENA, la participation à un contre-sommet comme celui organisé à Cancún lors de la conférence ministérielle de l'OMC, ou encore l'appui marqué au mouvement néozapatiste, référence centrale de l'altermondialisme.

\section{Conclusion}

On a vu que l'environnementalisme social s'était construit sur la base de la formulation et de la mise en œuvre de modèles alternatifs de gestion des ressources naturelles et de développement en direction des communautés indigènes et paysannes mexicaines, qu'il avait évolué ensuite au cours des années 1990 sous l'effet de son institutionnalisation dans ou face à l'État mexicain, mais aussi sous l'effet de l'internationalisation des financements et des logiques de travail. Si l'influence internationale est importante, notamment dans la dernière période, on peut considérer l'environnementalisme social mexicain comme une forme locale et originale d'écologie politique qui a développé une pensée et des pratiques proprement mexicaines.

Le spectre des acteurs de l'environnementalisme social est sûrement beaucoup plus large qu'on ne l'a décrit, surtout si l'on considère qu'il repose sur l'ensemble des innombrables projets de développement rural à caractère environnemental à travers le pays. Si la base et les forces vives de l'environnementalisme social sont donc encore liées aux communautés paysannes et/ou indigènes, s'opposant à des modèles de développement destructeurs ou luttant en faveur de la foresterie communautaire, de «reservas comunitarias» ou du café et de l'agriculture biologiques, on constate qu'une certaine expertise militante, à la fois technique et politique, s'est aussi développée à travers des organisations aux perspectives plus idéologiques et moins localisées. L'environnementalisme social mexicain oscille donc entre le local et le mondial, mais aussi entre projets de développement, expertise technique et prises de position 
critiques. Beaucoup plus qu'un mouvement clairement coordonné, ce courant représente une nébuleuse d'initiatives et d'organisations. Mais au-delà de ces innombrables variations, il est caractérisé par une même vision «intégrale » du développement et un souci constant d'articuler les problématiques environnementales et politico-sociales.

JEAN FOYER est sociologue, chercheur à l'Institut des sciences de la communication du CNRS et l'auteur de Il était une fois la bio-révolution (PUF, 2010) et, avec Éric Léonard, de De la integración nacional al desarrollo sustentable (CEDRSSA \& IRD, 2011). DAVID DUMOULIN est sociologue, chercheur au Centre de recherche et de documentation des Amériques (CREDA, UMR 7227) et enseignant à l'Institut des hautes études de l'Amérique latine (IHEAL), Université Sorbonne Nouvelle Paris 3. Il a coordonné, avec Christian Gros, Le multiculturalisme "au concret». Un modèle latino-américain? (Presses de la Sorbonne Nouvelle, 2012) et, avec Marielle Pepin-Lehalleur, Agir-en-réseau. Modèle d'action ou catégorie d'analyse? (PUR, 2012). 